\title{
ON CHEBYSHEV POLYNOMIALS in the COMPLEX PLANE
}

\author{
V. V. ANDRIEVSKII \\ Department of Mathematical Sciences, Kent State University, \\ Kent, OH 44242, U.S.A. \\ email: andriyev@math.kent.edu
}

\begin{abstract}
The estimates of the uniform norm of the Chebyshev polynomials associated with a compact set $K$ in the complex plane are established. These estimates are exact (up to a constant factor) in the case where $K$ consists of a finite number of quasiconformal curves or arcs. The case where $K$ is a uniformly perfect subset of the real line is also studied.
\end{abstract}

\section{Introduction and main results}

Let $K \subset \mathbf{C}$ be a compact set in the complex plane $\mathbf{C}$ with a connected complement $\Omega:=\overline{\mathbf{C}} \backslash K$, where $\overline{\mathbf{C}}:=\mathbf{C} \cup\{\infty\}$. We assume that $\operatorname{cap}(K)>0$, where $\operatorname{cap}(K)$ denotes the logarithmic capacity of $K$ (see [22] - [24]). Denote by $T_{n}(z)=T_{n}(z, K), n \in \mathbf{N}:=\{1,2, \ldots\}$ the $n$-th Chebyshev polynomial associated with $K$, i.e., $T_{n}(z)=z^{n}+c_{n-1} z^{n-1}+\ldots+c_{0}, c_{k} \in \mathbf{C}$, is the (unique) monic polynomial which minimizes the supremum norm $\left\|T_{n}\right\|_{K}:=\sup _{z \in K}\left|T_{n}(z)\right|$ among all monic polynomials of the same degree.

It is well-known (see, for example, [23, Theorem 5.5.4 and Corollary 5.5.5]) that

$$
\left\|T_{n}\right\|_{K} \geq \operatorname{cap}(K)^{n} \quad \text { and } \quad \lim _{n \rightarrow \infty}\left\|T_{n}\right\|_{K}^{1 / n}=\operatorname{cap}(K) .
$$

We are interested in estimates from above for the quantity

$$
t_{n}(K):=\frac{\left\|T_{n}\right\|_{K}}{\operatorname{cap}(K)^{n}} .
$$

We refer the reader to [25]-[27], [29]-[32], [34], [8], [4] and many references therein for a comprehensive survey of this subject.

First, let $K$ consist of disjoint closed connected sets (continua) $K^{j}, j=1,2, \ldots, m$, i.e.,

$$
K=\cup_{j=1}^{m} K^{j} ; \quad K^{j} \cap K^{k}=\emptyset \text { for } j \neq k ; \quad \operatorname{diam}\left(K^{j}\right)>0 .
$$

Here

$$
\operatorname{diam}(S):=\sup _{z, \zeta \in S}|z-\zeta|, \quad S \subset \mathbf{C}
$$

Key words and phrases: Chebyshev polynomial, equilibrium measure, quasiconformal curve, uniformly perfect set.

2000 Mathematics Subject Classification: 30A10, 30C10, 30C62, 30E10 
Theorem 1 Under the above assumptions,

$$
t_{n}(K)=O(\log n) \quad \text { for } n \rightarrow \infty .
$$

If more information is known about the geometry of $K,(1.2)$ can be improved, for example, in the following way. A Jordan curve $L \subset \mathbf{C}$ is called quasiconformal (see [1] or [16, p. 100]) if for every $z_{1}, z_{2} \in L$,

$$
\operatorname{diam}\left(L\left(z_{1}, z_{2}\right)\right) \leq \beta_{L}\left|z_{2}-z_{1}\right|,
$$

where $L\left(z_{1}, z_{2}\right)$ is the smaller subarc of $L$ between $z_{1}$ and $z_{2}$, a constant $\beta_{L}>1$ depends only on $L$. Any subarc of a quasiconformal curve is called a quasiconformal arc.

Theorem 2 Let each $K^{j}$ in (1.1) be either a quasiconformal arc or a closed Jordan domain bounded by a quasiconformal curve. Then

$$
t_{n}(K)=O(1) \text { for } n \rightarrow \infty .
$$

The estimate (1.4) was proved by other methods in [34] and recently in [31] (for sufficiently smooth $\partial K^{j}$ ), in [32] (for piecewise sufficiently smooth $\partial K^{j}$ ), and in [4] (for quasismooth in the sense of Lavrentiev $\partial K^{j}$ ).

The question whether (1.4) does hold for a general continuum seems to be still open. In the Oberwolfach meeting (see [14] or [20, p. 365]) Pommerenke asked about an example for a continuum $K$ such that the sequence $\left\{t_{n}(K)\right\}$ is unbounded. It is mentioned in [20, p. 365] that "D. Wrase in Karlsruhe has shown that an example constructed by J. Clunie [9] for a different purpose has the required property". But we could not find the proof of this result.

Moreover, in the case where $K$ is a continuum, one of the major sources for estimates of $t_{n}(K)$ are Faber polynomials $F_{n}(z)=F_{n}(z, K)$ associated with $K$ (see [25], [27]). Gaier [11, Theorem 2], using the same example by Clunie, [9] has shown that there exist a continuum $K_{*}$ bounded by a quasiconformal curve with $\operatorname{cap}\left(K_{*}\right)=1$, a positive constant $\alpha$ and an infinite set $\Lambda \subset \mathbf{N}$ such that for the (monic) polynomial $F_{n}(z)=F_{n}\left(z, K_{*}\right)$ we have

$$
\left\|F_{n}\right\|_{K_{*}}>n^{\alpha}, \quad n \in \Lambda .
$$

Note that the first result of this kind (without the restriction on $K_{*}$ to be a quasidisk) was proved by Pommerenke [19].

Hence, Theorem 1 and Theorem 2 reveal the essential difference between the Chebyshev and the Faber polynomials. It is worth pointing out that the case of multiply connected $\Omega$ presents a more delicate problem (see for example [34]). 
Let now $K \subset \mathbf{R}$, where $\mathbf{R}$ is the real line, consist of an infinite number of components. According to [13, Theorem 4.4] in this case $\left\{t_{n}(K)\right\}$ can increase faster than any sequence $\left\{t_{n}\right\}$ satisfying $t_{n} \geq 1$ and $\lim _{n \rightarrow \infty}\left(\log t_{n}\right) / n=0$. Therefore, in order to have particular bounds for $t_{n}(K)$ some additional assumptions on $K$ are needed. We assume that $K$ is uniformly perfect, which according to Beardon and Pommerenke [6] means that there exists a constant $0<\gamma_{K}<1$ such that for $z \in K$,

$$
K \cap\left\{\zeta: \gamma_{K} r \leq|z-\zeta| \leq r\right\} \neq \emptyset, \quad 0<r<\operatorname{diam}(K) .
$$

The classical Cantor set is an example of a uniformly perfect set. Pommerenke [21] has shown that uniformly perfect sets can be described using a density condition in terms of the logarithmic capacity. Namely, $K$ is uniformly perfect if and only if there exists a constant $0<\lambda_{K}<1$ such that for $z \in K$,

$$
\operatorname{cap}(K \cap\{\zeta:|\zeta-z| \leq r\}) \geq \lambda_{K} r, \quad 0<r<\operatorname{diam}(K) .
$$

Note that sets satisfying (1.5) play a significant role in the solution of the inverse problem of the constructive theory of functions of a complex variable. We refer to [28] where they are called $c$-dense sets. Other interesting properties of the uniformly perfect sets can be found in [12, pp. 343-345].

Theorem 3 For a uniformly perfect set $K \subset \mathbf{R}$ there exists a constant $c=$ $c(K)>0$ such that

$$
t_{n}(K)=O\left(n^{c}\right) \text { for } n \rightarrow \infty .
$$

Following Carleson [7] we say that a compact set $K \subset \mathbf{R}$ is homogeneous if there is a constant $\eta_{K}>0$ such that for all $x \in K$,

$$
|K \cap(x-r, x+r)| \geq \eta_{K} r, \quad 0<r<\operatorname{diam}(K) .
$$

Here, $|S|$ is the linear measure (length) of a (Borel) set $S \subset \mathbf{C}$ (see [22, p. 129]). The Cantor sets of positive length are examples of homogeneous sets (see [18, p. 125]). Recently Christiansen, Simon, and Zinchenko [8] have shown that for the homogeneous subsets of the real line the term $O\left(n^{c}\right)$ in (1.6) can be replaced by $O(1)$. It is worth pointing out that there is a principal difference between the above mentioned classes of compact sets, i.e., $K$ is the Parreau-Widom set in the case of the homogeneous $K \subset \mathbf{R}$ and it is not, in general, the Parreau-Widom set in the case of the uniformly perfect $K$. See [8] for more details.

In what follows, we use the convention that $c, c_{1}, \ldots$ denote positive constants (different in different sections) that are either absolute or they depend only on $K$; otherwise, the dependence on other parameters is explicitly stated. For the nonnegative functions $a$ and $b$ we write $a \preceq b$ if $a \leq c b$, and $a \asymp b$ if $a \preceq b$ and $b \preceq a$ simultaneously. 
We also use the additional notation

$$
d(z, S):=\operatorname{dist}(\{z\}, S):=\inf _{\zeta \in S}|z-\zeta|, \quad z \in \mathbf{C}, S \subset \mathbf{C} .
$$

\section{The basic potential-theoretic functions}

Let $K$ be as in (1.1). Following Widom [34], we extend the concept of Faber polynomials to the case of compact sets with the finite number of connected components. Since in [34] all $\partial K^{j}$ are sufficiently smooth curves, we need to add some purely technical details. Denote by $g_{\Omega}\left(z, z_{0}\right), z, z_{0} \in \Omega$, the Green function for $\Omega$ with pole at $z_{0}$. It has a multiple-valued harmonic conjugate $\tilde{g}_{\Omega}\left(z, z_{0}\right)$. Thus, the analytic function

$$
\Phi_{\Omega}\left(z, z_{0}\right):=\exp \left(g_{\Omega}\left(z, z_{0}\right)+i \tilde{g}_{\Omega}\left(z, z_{0}\right)\right)
$$

is also multiple-valued. We write $g_{\Omega}(z), \tilde{g}_{\Omega}(z)$, and $\Phi_{\Omega}(z)$ in the case $z_{0}=\infty$.

Let

$$
\begin{gathered}
g_{\Omega}(z):=0, \quad z \in K \\
K_{s}:=\{z \in \mathbf{C}: g(z) \leq s\}, \Omega_{s}:=\overline{\mathbf{C}} \backslash K_{s}, \quad s>0 .
\end{gathered}
$$

Then for $z \in \Omega_{s}$,

$$
g_{\Omega_{s}}(z)=g_{\Omega}(z)-s, \Phi_{\Omega_{s}}(z)=e^{-s} \Phi_{\Omega}(z) .
$$

For $n \in \mathbf{N}$, if $\Phi_{\Omega}(z)^{n}$ is single-valued in $\Omega_{1 / n^{2}}$, we set

$$
W_{n}(z):=\Phi_{\Omega}(z)^{n}, \quad z \in \Omega_{1 / n^{2}} .
$$

If $\Phi_{\Omega}(z)^{n}$ is multiple-valued in $\Omega_{1 / n^{2}}$, then according to [34, pp. 159, 211] there exist $q \leq m-1$ points $z_{1, n}, \ldots, z_{q, n} \in \Omega_{1 / n^{2}}$ such that the function

$$
W_{n}(z):=\Phi_{\Omega}(z)^{n} \prod_{l=1}^{q} \Phi_{\Omega_{1 / n^{2}}}\left(z, z_{l, n}\right)^{-1}, \quad z \in \Omega_{1 / n^{2}}
$$

is single-valued in $\Omega_{1 / n^{2}}$. Moreover, all $z_{l, n}$ lie in the convex hull of $K_{1 / n^{2}}$.

In both cases we consider the entire function

$$
F_{n}(z):=\frac{1}{2 \pi i} \int_{C_{n}} \frac{W_{n}(\zeta)}{\zeta-z} d \zeta, \quad z \in \mathbf{C},
$$

where $C_{n} \subset \Omega_{1 / n^{2}} \backslash\{\infty\}$ is a Jordan curve, oriented in the positive direction, containing $K_{1 / n^{2}}$ and $z$ in its interior. 
Since all points $z_{l, n}$ are in the convex hull of $K_{1}$, by the symmetry property of the Green function, we obtain

$$
\begin{aligned}
\left|\prod_{l=1}^{q} \Phi_{\Omega_{1 / n^{2}}}\left(\infty, z_{l, n}\right)\right| & =\exp \left(\sum_{l=1}^{q} g_{\Omega_{1 / n^{2}}}\left(z_{l, n}\right)\right) \\
& \leq \exp \left(\sum_{l=1}^{q} g_{\Omega}\left(z_{l, n}\right)\right) \preceq 1 .
\end{aligned}
$$

For $z \in \Omega_{1 / n^{2}} \backslash\{\infty\}$, let $C_{n}^{\prime} \subset \Omega_{1 / n^{2}} \backslash\{\infty\}$ be any Jordan curve, oriented in the positive direction, containing $K_{1 / n^{2}}$ in its interior and $z$ in its exterior. Since by the Cauchy formula

$$
F_{n}(z)=W_{n}(z)+\frac{1}{2 \pi i} \int_{C_{n}^{\prime}} \frac{W_{n}(\zeta)}{\zeta-z} d \zeta
$$

we see that $F_{n}(z)=\alpha_{n} z^{n}+\ldots$ is a polynomial with the property

$$
\begin{aligned}
\left|\alpha_{n}\right| & =\lim _{z \rightarrow \infty}\left|\frac{F_{n}(z)}{z^{n}}\right|=\lim _{z \rightarrow \infty}\left|\frac{W_{n}(z)}{z^{n}}\right| \\
& =\operatorname{cap}(K)^{-n} \prod_{l=1}^{q}\left|\Phi_{\Omega_{1 / n^{2}}}\left(z_{l, n}\right)\right|^{-1} \succeq \operatorname{cap}(K)^{-n} .
\end{aligned}
$$

Now let $K$ consist of one component, i.e., $m=1$ and let $\Phi_{\Omega}: \Omega \rightarrow \mathbf{D}^{*}:=\{w$ : $|w|>1\}$ be the Riemann conformal mapping with $\Phi_{\Omega}(\infty)=\infty, \Phi_{\Omega}^{\prime}(\infty)>0$. We follow a technique of [10, Chapter IX], [5, p. 387] and for $k, n \in \mathbf{N}, k \geq 2$, consider the Dzjadyk polynomial kernel

$$
K_{1,1, k, n}(\zeta, z), \quad \zeta \in \Omega \backslash\{\infty\}, z \in K
$$

which is a polynomial with respect to $z$ of degree $(k+3) k(n-1)-1$ with continuous coefficients depending on $\zeta$.

According to $[5$, p. 389, Theorem 2.4] we have

$$
\left|\frac{1}{\zeta-z}-K_{1,1, k, n}(\zeta, z)\right| \leq c_{1} \frac{\left|\tilde{\zeta}_{1 / n}-\zeta\right|^{k}}{|\zeta-z|\left|\tilde{\zeta}_{1 / n}-z\right|^{k}}\left(1+\left|\frac{\zeta-z}{\tilde{\zeta}_{1 / n}-z}\right|\right)^{k}
$$

where $c_{1}=c_{1}(K, k)$ and

$$
\tilde{\zeta}_{\delta}:=\Phi_{\Omega}^{-1}\left((1+\delta) \Phi_{\Omega}(\zeta)\right), \quad \delta>0
$$

Let $w:=\Phi_{\Omega}(\zeta), \tilde{w}_{1 / n}:=w(1+1 / n)$. A straightforward calculation shows that for $n>32^{2}$ and $|w| \geq 1+32 / n$, we have

$$
\frac{\left|\tilde{w}_{1 / n}-w\right|}{\left|\tilde{w}_{1 / n}\right|-1}<\frac{1}{32}
$$


Therefore, [5, p. 23, Lemma 2.3] implies

$$
\frac{\left|\tilde{\zeta}_{1 / n}-\zeta\right|}{\left|\tilde{\zeta}_{1 / n}-z\right|} \leq 16 \frac{\left|\tilde{w}_{1 / n}-w\right|}{\left|\tilde{w}_{1 / n}\right|-1}<\frac{1}{2},
$$

i.e.,

$$
\left|\frac{1}{\zeta-z}-K_{1,1, k, n}(\zeta, z)\right| \leq c_{2} \frac{d(\zeta, K)^{k}}{|\zeta-z|^{k+1}}, \quad c_{2}=c_{2}(K, k) .
$$

We summarize our reasoning as follows. Given $k \in \mathbf{N}$, there exist sufficiently large constants $n_{0}=n_{0}(k)$ and $c_{3}=c_{3}(k)$ such that for any integer $n>n_{0}$ and $\zeta$ with $\left|\Phi_{\Omega}(\zeta)\right|-1 \geq c_{3} / n$, there exists a polynomial

$$
p_{n, k, \zeta, K}(z)=\sum_{l=0}^{n} a_{l, k, K}(\zeta) z^{l},
$$

where $a_{l, k, K}$ are continuous functions of $\zeta$, satisfying

$$
\left|\frac{1}{\zeta-z}-p_{n, k, \zeta, K}(z)\right| \leq c_{2} \frac{d(\zeta, K)^{k}}{|\zeta-z|^{k+1}}, \quad z \in K .
$$

Indeed, to get (2.4) we can take

$$
p_{n, k, \zeta, K}(z):=K_{1,1, k, N}(\zeta, z), \quad N:=\left\lfloor\frac{n}{k(k+3)}\right\rfloor .
$$

Furthermore, by virtue of (2.3), for $\zeta \in \Omega$ with $c_{4} \leq\left|\Phi_{\Omega}(\zeta)\right|-1 \leq c_{5}$ we have

$$
\left|\frac{1}{\zeta-z}-p_{n, k, \zeta, K}(z)\right| \leq \frac{c_{6}}{n^{k}}, \quad z \in K, c_{6}=c_{6}\left(c_{4}, c_{5}, K, k\right) .
$$

Let $K$ now be as in (1.1) with $m>1$. Denote by $r_{K}>0$ any fixed number such that $K_{r_{K}}$ consists of exactly $m$ components, i.e.,

$$
K_{r_{K}}=\cup_{j=1}^{m} K_{r_{K}}^{j}, \quad K^{j} \subset K_{r_{K}}^{j} .
$$

Let $\Omega^{j}:=\overline{\mathbf{C}} \backslash K^{j}$. The maximum principle for the appropriate linear combination of harmonic functions $g_{\Omega}$ and $\log \left|\Phi_{\Omega^{j}}\right|$ in $K_{r_{K}}^{j} \backslash K^{j}$ shows that

$$
g_{\Omega}(\zeta) \preceq \log \left|\Phi_{\Omega^{j}}(\zeta)\right|, \quad \zeta \in K_{r_{K}}^{j} \backslash K^{j} .
$$

For sufficiently large $v \in \mathbf{N}, \zeta \in K_{r_{K}}^{j} \backslash K^{j}$ with $\left|\Phi_{\Omega^{j}}(\zeta)\right|-1 \geq c_{3} / v$, and $z \in K^{l}, l=1, \ldots m$, by virtue of (2.4) and (2.5), applied for the continuum $K^{l}$, we have

$$
\left|\frac{1}{\zeta-z}-p_{v, k, \zeta, K^{l}}(z)\right| \leq c_{7} \begin{cases}\frac{d\left(\zeta, K^{j}\right)^{k}}{|\zeta-z|^{k+1}} & \text { if } l=j \\ n^{-k} & \text { if } l \neq j .\end{cases}
$$


Here $c_{7}:=c_{2}+c_{6}$.

For $\zeta$ as in $(2.7)$ and $l=1, \ldots, m$, consider functions

$$
\begin{gathered}
h_{l}(z):= \begin{cases}1 & \text { if } z \in K^{l}, \\
0 & \text { if } z \in K \backslash K^{l},\end{cases} \\
f_{\zeta, l}(z):=\frac{h_{l}(z)}{\zeta-z}, \quad z \in K,
\end{gathered}
$$

so that

$$
\frac{1}{\zeta-z}=\sum_{l=1}^{m} f_{\zeta, l}(z), \quad z \in K .
$$

Since $h_{l}$ can be extended analytically to $K_{r_{K}}$, by the Walsh approximation theorem [33, pp. 75-76] there is $u_{0}=u_{0}(K) \in \mathbf{N}$, such that for any integer $u>u_{0}$, there exists a polynomial $q_{u, l} \in \mathbf{P}_{u}$ satisfying

$$
|| h_{l}-q_{u, l} \|_{K} \leq e^{-u r_{K}} .
$$

For sufficiently large $n$ and $\zeta \in K_{r_{K}}^{j} \backslash K^{j}$ with $\left|\Phi_{\Omega^{j}}(\zeta)\right|-1 \geq c^{*} / n \geq c_{3} / v$, where $v$ and the constant $c^{*}$ are to be chosen later, consider the polynomial

$$
t_{u, v, k, \zeta, l}:=q_{u, l} p_{v, k, \zeta, K^{l}} \in \mathbf{P}_{u+v} .
$$

Let

$$
R_{K}:=\max _{1 \leq j \leq m} \| \log \left|\Phi_{\Omega^{j}}\right|||_{\partial K} .
$$

Since for $z \in K^{p}, p=1, \ldots, m$,

$\left|f_{\zeta, l}(z)-t_{u, v, k, \zeta, l}(z)\right| \leq \begin{cases}\left|f_{\zeta, l}(z)-p_{v, k, \zeta, K^{l}}(z)\right|+\left|p_{v, k, \zeta, K^{l}}(z)\right|\left|h_{l}(z)-q_{u, l}(z)\right| & \text { if } p=l, \\ \left|p_{v, k, \zeta, K^{l}}(z)\right|\left|h_{l}(z)-q_{u, l}(z)\right| & \text { if } p \neq l,\end{cases}$

by (2.7), (2.8), and the Bernstein-Walsh lemma (see [33, p. 77] or [24, p. 153]), we obtain the following estimates:

if $l=j$, then

$$
\left|f_{\zeta, l}(z)-t_{u, v, k, \zeta, l}(z)\right| \leq \begin{cases}c_{7} \frac{d\left(\zeta, K^{j}\right)^{k}}{|\zeta-z|^{k+1}}+\frac{c_{7}+1}{d\left(\zeta, K^{j}\right)} e^{-u r_{K}} & \text { if } p=l \\ \frac{c_{7}+1}{d\left(\zeta, K^{j}\right)} e^{v R_{K}-u r_{K}} & \text { if } p \neq l\end{cases}
$$


if $l \neq j$, then

$$
\leq \begin{cases}\frac{c_{7}}{n^{k}}+c_{8} e^{-u r_{K}} & \text { if } p=l, \\ c_{7} \frac{d\left(\zeta, K^{j}\right)^{k}}{|\zeta-z|^{k+1}}+\frac{c_{7}+1}{d\left(\zeta, K^{j}\right)} e^{-u r_{K}} & \text { if } p=j, \\ c_{8} e^{v R_{K}-u r_{K}} & \text { if } p \neq l, p \neq j .\end{cases}
$$

Therefore, for the polynomial

$$
t_{u, v, k, \zeta}:=\sum_{l=1}^{m} t_{u, v, k, \zeta, l} \in \mathbf{P}_{u+v}
$$

according to (2.9) and (2.10) for $\zeta$ as in (2.7) and $z \in K^{p}$, we obtain:

if $p=j$, then

$$
\begin{aligned}
& \left|\frac{1}{\zeta-z}-t_{u, v, k, \zeta}(z)\right| \\
\leq & m\left(c_{7} \frac{d\left(\zeta, K^{j}\right)^{k}}{|\zeta-z|^{k+1}}+\frac{c_{7}+1}{d\left(\zeta, K^{j}\right)} e^{-u r_{K}}\right) ;
\end{aligned}
$$

if $p \neq j$, then

$$
\begin{aligned}
\left|\frac{1}{\zeta-z}-t_{u, v, k, \zeta}(z)\right| \leq & \frac{c_{7}+1}{d\left(\zeta, K^{j}\right)} e^{v R_{K}-u r_{K}} \\
& +\frac{c_{7}}{n^{k}}+c_{8} e^{-u r_{K}}+(m-2) c_{8} e^{v R_{K}-u r_{K}} .
\end{aligned}
$$

Let

$$
u:=\left\lfloor\frac{2 R_{K}(n-1)}{2 R_{K}+r_{K}}\right\rfloor, \quad v:=\left\lfloor\frac{r_{K}(n-1)}{2 R_{K}+r_{K}}\right\rfloor
$$

Note that $v \geq n / c_{9}$. To be sure that (2.11) and (2.12) hold we need to have $c_{3} / v \leq c^{*} / n$ which dictates the choice $c^{*}:=c_{3} c_{9}$.

Thus, using the Löwner inequality (see [5, p. 359, Corollary 2.5]), $d\left(\zeta, K^{j}\right) \geq$ $c_{10} / n^{2}, c_{10}=c_{10}(K, k)$, we obtain a polynomial

$$
s_{n-1, k, \zeta}:=t_{u, v, k, \zeta} \in \mathbf{P}_{n-1}
$$

satisfying, by virtue of (2.11) and (2.12), for $\zeta \in K_{r_{K}}^{j} \backslash K^{j}$ with $\left|\Phi_{\Omega^{j}}(\zeta)\right|-1 \geq$ $c^{*} / n$, where $c^{*}=c^{*}(K, k)$ and $n>n_{1}=n_{1}(K, k)$, the inequality

$$
\left|\frac{1}{\zeta-z}-s_{n-1, k, \zeta}(z)\right| \leq c_{11} \begin{cases}\frac{d\left(\zeta, K^{j}\right)^{k}}{|\zeta-z|^{k+1}} & \text { if } z \in K^{j}, \\ n^{-k} & \text { if } z \in K \backslash K^{j},\end{cases}
$$


where $c_{11}=c_{11}(K, k)$.

\section{Chebyshev polynomials for a system of continua}

We start with the proof of the following estimate.

Lemma 1 Let $K$ be as in (1.1). Then for $k \in \mathbf{N}$,

$$
t_{n}(K) \leq c_{1} \sum_{j=1}^{m}\left\|\int_{L_{c^{*} / n, j}} \frac{d\left(\zeta, K^{j}\right)^{k}|d \zeta|}{|\zeta-\cdot|^{k+1}}\right\|_{K^{j}}, \quad n \geq n_{1},
$$

where $c^{*}$ and $n_{1}$ are the constants from (2.13), $c_{1}=c_{1}(K, k)$, and

$$
L_{\delta, j}:=\left\{\zeta \in \Omega^{j}:\left|\Phi_{\Omega^{j}}(\zeta)\right|=1+\delta\right\}, \quad \delta>0 .
$$

Proof. Let $F_{n}$ be defined by (2.1). By our assumption $n$ is so large that the curves $S_{n, j}:=L_{c^{*} / n, j} \subset \Omega_{1 / n^{2}}$ are mutually disjoint. Let $S_{n}:=\cup_{j=1}^{m} S_{n, j}$.

By $\left[5\right.$, p. 23, Lemma 2.3], for $\zeta \in \Omega^{j}, w:=\Phi_{\Omega^{j}}(\zeta)$, and $\Psi_{\Omega^{j}}:=\Phi_{\Omega^{j}}^{-1}$, we have

$$
\left|\Psi_{\Omega^{j}}^{\prime}(w)\right| \asymp \frac{d\left(\zeta, K^{j}\right)}{|w|-1} .
$$

Therefore,

$$
\begin{aligned}
\left|S_{n, j}\right| & =\int_{|w|=1+c^{*} / n}\left|\Psi_{\Omega^{j}}^{\prime}(w)\right||d w| \\
& \asymp \frac{n}{c^{*}} \int_{|w|=1+c^{*} / n} d\left(\Psi_{\Omega^{j}}(w), K^{j}\right)|d w| \leq c n, \quad c=c(K, k) .
\end{aligned}
$$

By the Cauchy formula

$$
F_{n}(z)=\frac{1}{2 \pi i} \sum_{j=1}^{m} \int_{S_{n, j}} \frac{W_{n}(\zeta)}{\zeta-z} d \zeta, \quad z \in K
$$

We can certainly assume that $k>1$. Consider polynomial $F_{n}^{*}(z)=\alpha_{n} z^{n}+\ldots \in$ $\mathbf{P}_{n}$ defined as follows

$$
F_{n}^{*}(z):=\frac{1}{2 \pi i} \sum_{j=1}^{m} \int_{S_{n, j}} W_{n}(\zeta)\left(\frac{1}{\zeta-z}-s_{n-1, k, \zeta}(z)\right) d \zeta, \quad z \in K
$$

where $s_{n-1, k, \zeta} \in \mathbf{P}_{n-1}$ satisfies (2.13). 
Since by (2.6), for $\zeta \in S_{n}$,

$$
\left|W_{n}(\zeta)\right| \leq\left|\Phi_{\Omega}(\zeta)\right|^{n}=\exp \left(n g_{\Omega}(\zeta)\right) \leq c_{2},
$$

where $c_{2}=c_{2}(K, k)$, according to (2.13) and (3.3), for $z \in K^{j}$, we obtain

$$
\begin{aligned}
\left|F_{n}^{*}(z)\right| & \leq c_{3}\left(\int_{S_{n, j}} \frac{d\left(\zeta, K^{j}\right)^{k}}{|\zeta-z|^{k+1}}|d \zeta|+n^{-k} \sum_{l=1, l \neq j}^{m}\left|S_{n, l}\right|\right) \\
& \leq c_{4} \int_{S_{n, j}} \frac{d\left(\zeta, K^{j}\right)^{k}}{|\zeta-z|^{k+1}}|d \zeta|,
\end{aligned}
$$

where $c_{l}=c_{l}(K, k), l=3,4$.

Making use of (2.2) and the obvious inequality $t_{n}(K) \operatorname{cap}(K)^{n} \leq|| F_{n}^{*}||_{K} /\left|\alpha_{n}\right|$ we finally obtain (3.1).

Proof of Theorem 1. Changing the variable in the integrals from (3.1) and using (3.2), for sufficiently large $n$, we obtain

$$
\begin{aligned}
& \left\|\int_{L_{c^{*} / n, j}} \frac{d\left(\zeta, K^{j}\right)|d \zeta|}{|\zeta-\cdot|^{2}}\right\|_{K^{j}} \\
\asymp & \frac{1}{n}\left\|\int_{|w|=1+c^{*} / n}\left(\frac{\left|\Psi_{\Omega^{j}}^{\prime}(w)\right|}{\left|\Psi_{\Omega^{j}}(w)-\cdot\right|}\right)^{2}|d w|\right\|_{K^{j}} .
\end{aligned}
$$

Furthermore, since by [27, Chapter IX, §4, Lemma 3],

$$
\left\|\int_{|w|=1+c^{*} / n}\left(\frac{\left|\Psi_{\Omega^{j}}^{\prime}(w)\right|}{\left|\Psi_{\Omega^{j}}(w)-\cdot\right|}\right)^{2}|d w|\right\|_{K^{j}} \preceq n \log n,
$$

the inequalities (3.1) (with $k=1$ ) and (3.4) imply (1.2).

Theorem 2 is a particular case of a more general result which we describe below. Let $K$ consist of one component, i.e., $m=1$, and let $\Omega$ be a John domain which can be defined as follows (see [22, p. 98]). For a crosscut $\gamma \subset \Omega \backslash\{\infty\}$ of $\Omega$ let $H(\gamma)$ be a bounded component of $\Omega \backslash \gamma$. We say that $\gamma$ is a circular crosscut if $\gamma \subset \Omega \cap C(z, r)$ for some $z \in \partial \Omega=\partial K, r>0$, and $z \in \overline{H(\gamma)}$. Here $C(z, r):=\{\zeta:|\zeta-z|=r\}$. Then $\Omega$ is a John domain if there exists a constant $\lambda_{\Omega}>1$ such that for any circular crosscut $\gamma$ of $\Omega$,

$$
\operatorname{diam}(H(\gamma)) \leq \lambda_{\Omega}|\gamma| .
$$


By virtue of (1.3) the complement of a quasiconformal arc as well as the unbounded Jordan domain with a quasiconformal boundary both are John domains.

According to (3.5) the function $\Psi_{\Omega}$ has a continuous extension to $\overline{\mathrm{D}^{*}}$ which we denote by the same letter $\Psi_{\Omega}$. Next, we assume that $\partial K$ is piecewise quasiconformal, i.e., there exist

$$
\theta_{1}<\theta_{2}<\ldots<\theta_{p}<\theta_{p+1}:=\theta_{1}+2 \pi, \quad p \geq 2
$$

such that each $J_{l}:=\Psi_{\Omega}\left(J_{l}^{\prime}\right), l=1, \ldots, p$, where $J_{l}^{\prime}:=\left\{e^{i \theta}: \theta_{l} \leq \theta \leq \theta_{l+1}\right\}$ is a quasiconformal arc.

Let

$$
\begin{gathered}
z_{l}:=\Psi_{\Omega}\left(e^{i \theta_{j}}\right), \quad \Gamma_{l}^{\prime}:=\left\{r e^{i \theta_{j}}: r \geq 1\right\}, \quad \Gamma_{l}:=\Psi_{\Omega}\left(\Gamma_{l}^{\prime}\right), \\
\Omega_{l}^{\prime}:=\left\{r e^{i \theta}: \theta_{l}<\theta<\theta_{l+1}, r>1\right\}, \quad \Omega_{l}:=\Psi_{\Omega}\left(\Omega_{l}^{\prime}\right) .
\end{gathered}
$$

By [3, Lemma 2],

$$
\left|\zeta-z_{l}\right| \preceq d(\zeta, K), \quad \zeta \in \Gamma_{l} .
$$

Moreover, according to $[3,(4.14)]$,

$$
\left|\Gamma_{l}\left(\zeta_{1}, \zeta_{2}\right)\right| \preceq\left|\zeta_{2}-\zeta_{1}\right|, \quad \zeta_{1}, \zeta_{2} \in \Gamma_{l} .
$$

Here for any arc or unbounded curve $\Gamma \subset \mathbf{C}$ and $\zeta_{1}, \zeta_{2} \in \Gamma$, we denote by $\Gamma\left(\zeta_{1}, \zeta_{2}\right)$ the bounded subarc of $\Gamma$ between these points.

Thus, by virtue of (3.6) and (3.7), the curve $L_{l}^{*}:=\partial \Omega_{l}=\Gamma_{l} \cup J_{l} \cup \Gamma_{l+1}$ satisfies

$$
\operatorname{diam}\left(L_{l}^{*}\left(\zeta_{1}, \zeta_{2}\right)\right) \preceq\left|\zeta_{2}-\zeta_{1}\right|, \quad \zeta_{1}, \zeta_{2} \in L_{l}^{*}
$$

i.e., by the Ahlfors criterion (see [16, p. 100]), $L_{l}^{*}$ is quasiconformal. Since by the same Ahlfors criterion $\partial \Omega_{l}^{\prime}=\Gamma_{l}^{\prime} \cup J_{l}^{\prime} \cup \Gamma_{l+1}^{\prime}$ is also quasiconformal, the restriction of $\Phi_{\Omega}$ to $\Omega_{l}$ can be extended to a $Q_{l}$-quasiconformal homeomorphism $\Phi_{l}: \mathbf{C} \rightarrow \mathbf{C}$ with some $Q_{l} \geq 1$ (see [16, p. 98]).

The following result describes the distortion properties of $\Phi_{l}$ and the inverse mapping $\Phi_{l}^{-1}$ which both are $Q$-quasiconformal with $Q:=\max _{l=1, \ldots, p} Q_{l}$.

Lemma 2 ([5, p. 29]) Let $F: \overline{\mathbf{C}} \rightarrow \overline{\mathbf{C}}$ be a $Q$-quasiconformal mapping, $Q \geq 1$, with $F(\infty)=\infty$. Let $\zeta_{k} \in \mathbf{C}, w_{k}:=F\left(\zeta_{k}\right), k=1,2,3$, be such that $\left|w_{1}-w_{2}\right| \leq$ $c_{5}\left|w_{1}-w_{3}\right|$. Then $\left|\zeta_{1}-\zeta_{2}\right| \leq c_{6}\left|\zeta_{1}-\zeta_{3}\right|$ and, in addition,

$$
\frac{1}{c_{7}}\left|\frac{w_{1}-w_{3}}{w_{1}-w_{2}}\right|^{1 / Q} \leq\left|\frac{\zeta_{1}-\zeta_{3}}{\zeta_{1}-\zeta_{2}}\right| \leq c_{7}\left|\frac{w_{1}-w_{3}}{w_{1}-w_{2}}\right|^{Q},
$$

where $c_{j}=c_{j}\left(c_{5}, Q\right), j=6,7$. 
We claim that for $z \in \partial K \backslash J_{l}$,

$$
d\left(z, J_{l}\right) \preceq d\left(z, \Omega_{l}\right) .
$$

Indeed, let $z_{l}^{\prime} \in \partial \Omega_{l}$ be such that $\left|z-z_{l}^{\prime}\right|=d\left(z, \Omega_{l}\right)$. The nontrivial case arises when $z_{l}^{\prime} \notin J_{l}$, i.e., $z_{l}^{\prime} \in \Gamma_{k}$ for $k=l$ or $k=l+1$. Then by (3.6) we obtain

$$
d\left(z, J_{l}\right) \leq\left|z-z_{k}\right| \leq\left|z-z_{l}^{\prime}\right|+\left|z_{l}^{\prime}-z_{k}\right| \preceq\left|z-z_{l}^{\prime}\right|=d\left(z, \Omega_{l}\right),
$$

which yields (3.8).

For $z \in \partial K$, denote by $z_{l}^{*}$ any point of $J_{l}$ with the property $\left|z-z_{l}^{*}\right|=d\left(z, J_{l}\right)$. We claim that

$$
\left|\zeta-z_{l}^{*}\right| \preceq|\zeta-z|, \quad \zeta \in \Omega_{l}, z \in \partial K \backslash J_{l} .
$$

Indeed, by (3.8),

$$
\left|\zeta-z_{l}^{*}\right| \leq|\zeta-z|+\left|z-z_{l}^{*}\right| \preceq|\zeta-z|+d\left(z, \Omega_{l}\right) \leq 2|\zeta-z|
$$

and (3.9) follows.

For $\zeta \in \Omega \backslash\{\infty\}$ denote by $\zeta_{K}:=\Psi_{\Omega}\left(\Phi_{\Omega}(\zeta) /\left|\Phi_{\Omega}(\zeta)\right|\right)$ the "projection" of $\zeta$ on $K$. As an immediate application of Lemma 2, for $\zeta \in \Omega_{l}$ and $z \in J_{l}$, we have

$$
\frac{d(\zeta, K)}{|\zeta-z|} \leq\left|\frac{\zeta-\zeta_{K}}{\zeta-z}\right| \preceq\left(\frac{\left|\Phi_{l}(\zeta)\right|-1}{\left|\Phi_{l}(\zeta)-\Phi_{l}(z)\right|}\right)^{1 / Q} .
$$

Now let $K$ be as in (1.1). We assume that each $\Omega^{j}$ is a John domain and each $\partial K^{j}$ is piecewise quasiconformal, i.e., each $\partial K^{j}$ consists of $p_{j}$ quasiconformal $\operatorname{arcs} J_{l, j}, l=1, \ldots, p_{j}$ as described above. Let $\Phi_{l, j}$ be the appropriate quasiconformal homeomorphism of $\mathbf{C}$ which is conformal in a subdomain $\Omega_{l}^{j}$ of $\Omega^{j}$ with $J_{l, j} \subset \partial \Omega_{l}^{j}$. For $z \in \partial K^{j}$, denote by $z_{l, j}^{*}$ the nearest to $z$ point of $J_{l, j}$ and let $w_{l, j}:=\Phi_{l, j}\left(z_{l, j}^{*}\right), J_{l, j}^{\prime}:=\Phi_{l, j}\left(J_{l, j}\right)$.

According to (3.2), Lemma 2 with $F=\Psi_{\Omega^{j}}$ restricted to $\Phi_{\Omega^{j}}\left(\Omega_{l}^{j}\right)$ and the triplet of points $\tau, \tau /|\tau|, w_{l, j}$, as well as (3.9), for $z \in \partial K^{j}, s=c^{*} / n$, and sufficiently large $n$, we obtain

$$
\begin{aligned}
\int_{L_{s, j}} \frac{d(\zeta, K)^{k}|d \zeta|}{|\zeta-z|^{k+1}} & =\sum_{l=1}^{p_{j}} \int_{L_{s, j} \cap \Omega_{l}^{j}} \frac{d(\zeta, K)^{k}|d \zeta|}{|\zeta-z|^{k+1}} \preceq \sum_{l=1}^{p_{j}} \int_{L_{s, j} \cap \Omega_{l}^{j}} \frac{d(\zeta, K)^{k}|d \zeta|}{\left|\zeta-z_{l, j}^{*}\right|^{k+1}} \\
& \preceq \sum_{l=1}^{p_{j}} \frac{1}{s} \int_{|\tau|=1+s, \tau /|\tau| \in J_{l, j}^{\prime}}\left|\frac{\Psi_{\Omega^{j}}(\tau)-\Psi_{\Omega^{j}}(\tau /|\tau|)}{\Psi_{\Omega^{j}}(\tau)-\Psi_{\Omega^{j}}\left(w_{l, j}\right)}\right|^{k+1}|d \tau| \\
& \preceq \sum_{l=1}^{p_{j}} \frac{1}{s} \int_{|\tau|=1+s} \frac{s^{(k+1) / Q}|d \tau|}{\left|\tau-w_{l, j}\right|^{(k+1) / Q}} \preceq 1
\end{aligned}
$$


if we fix $k$ satisfying $k+1>Q$.

Comparing the last estimate with Lemma 1 we obtain the following statement.

Theorem 4 Let $K$ be as in (1.1). Assume that each $\Omega^{j}$ is a John domain and each $\partial K^{j}$ is piecewise quasiconformal. Then (1.4) holds.

This theorem yields Theorem 2 .

\section{Chebyshev polynomials for uniformly perfect sets}

We introduce some definitions and notations from geometric function theory. Let $K \subset \mathbf{R}$ be a uniformly perfect set satisfying

$$
K \subset I:=[-1,1], \pm 1 \in K \neq I .
$$

The open (with respect to $\mathbf{R}$ ) set $I \backslash K$ consists of either a finite number $N \geq 1$ or an infinite number $N=\infty$ of disjoint open intervals, i.e.,

$$
I \backslash K=\bigcup_{j=1}^{N}\left(\alpha_{j}, \beta_{j}\right),
$$

where $\left(\alpha_{j}, \beta_{j}\right) \cap\left(\alpha_{k}, \beta_{k}\right)=\emptyset$ for $j \neq k$.

It follows immediately from (1.5) that $\Omega$ is regular (for the Dirichlet problem), see [23], [24], i.e., $g_{\Omega}$ extends continuously to $K$ and $g_{\Omega}(x):=0, x \in K$. Moreover, the Green function satisfies

$$
g_{\Omega}(\zeta) \leq c_{1} d(\zeta, K)^{\alpha}, \quad \zeta \in \mathbf{C}
$$

where constants $c_{1}$ and $\alpha$ could depend only on $\lambda_{K}$ from (1.5), see [15, Lemma 4.1] or [12, p. 119].

We need the Levin conformal mapping which can be defined as follows (for details, see [17], [2]). Consider the univalent in the upper half-plane $\mathbf{H}:=\{z$ : $\Im z>0\}$ function

$$
\phi(z)=\phi(z, K):=\pi+i\left(\int_{K} \log (z-\zeta) d \mu(\zeta)-\log \operatorname{cap}(K)\right), \quad z \in \mathbf{H},
$$

where $\mu=\mu_{K}$ is the equilibrium measure for $K$. It maps $\mathbf{H}$ onto a vertical half-strip with $N$ slits parallel to the imaginary axis, i.e., the domain

$$
\Sigma_{K}:=\{w: 0<\Re w<\pi, \Im w>0\} \backslash \bigcup_{j=1}^{N}\left[u_{j}, u_{j}+i v_{j}\right],
$$


where $0<u_{j}=u_{j}(K)<\pi$ and $v_{j}=v_{j}(K)>0$.

The continuous extension of $\phi$ to $\overline{\mathbf{H}}$ satisfies the following boundary correspondence

$$
\begin{gathered}
\phi(\infty)=\infty, \phi((-\infty,-1])=\{w: \Re w=0, \Im w \geq 0\}, \\
\phi([1, \infty))=\{w: \Re w=\pi, \Im w \geq 0\}, \quad \phi(K)=[0, \pi], \\
\phi\left(\left[\alpha_{j}, \beta_{j}\right]\right)=\left[u_{j}, u_{j}+i v_{j}\right], \quad j=1, \ldots, N .
\end{gathered}
$$

Note that in the last relation each point of $\left[u_{j}, u_{j}+i v_{j}\right)$ has two preimages on $\left[\alpha_{j}, \beta_{j}\right]$.

The crucial fact is that $\phi$ satisfies

$$
g_{\Omega}(z)=\Im\{\phi(z)\}, \quad z \in \overline{\mathbf{H}} .
$$

For a horizontal crosscut $\gamma$ of $\Sigma_{K}$, i.e., an interval $\gamma=(a+i b, c+i b) \subset \Sigma_{k}$ with endpoints on $\partial \Sigma_{K}$, denote by $h(\gamma)$ its "height", that is, $h(\gamma):=b$.

Lemma 3 Any horizontal crosscut $\gamma$ of $\Sigma_{K}$ with the property $h(\gamma) \leq \sup _{j} v_{j}$ satisfies

$$
h(\gamma) \leq c_{2}|\gamma|, \quad c_{2}=c_{2}\left(\lambda_{K}\right)
$$

Proof. For convenience, let $u_{-1}:=0$ and $u_{0}:=\pi$. Let $\gamma=\left(u_{j}+i h(\gamma), u_{k}+\right.$ $i h(\gamma)$ ) and $R:=\left\{w=u+i v: u_{j}<u<u_{k}, 0<v<h(\gamma)\right\}$. Denote by $\Gamma^{\prime}$ the family of crosscuts of $\Sigma_{K} \cap R$ which join $\left(u_{j}, u_{k}\right)$ to $\gamma$ and let $\Gamma^{*}$ be the family of crosscuts of the rectangle $R$ which join its horizontal boundary intervals. We refer to [1], [16], [12] for the basic properties of the module of a family of curves and arcs (such as conformal invariance, comparison principle, composition laws, etc.) We use these properties without further citation.

For the modules of $\Gamma^{\prime}$ and $\Gamma^{*}$ we have

$$
m\left(\Gamma^{\prime}\right) \leq m\left(\Gamma^{*}\right)=\frac{|\gamma|}{h(\gamma)} .
$$

At the same time, we claim that for the module of $\Gamma:=\phi^{-1}\left(\Gamma^{\prime}\right)$ the estimate

$$
m(\Gamma) \geq c_{3}, \quad c_{3}=c_{3}\left(\lambda_{K}\right)
$$

holds.

Indeed, without loss of generality, we assume that $j, k \geq 1$ and $\beta_{j}-\alpha_{j} \leq$ $\beta_{k}-\alpha_{k}$. The other particular cases may be handled in much the same way. Denote by $\Gamma_{1}$ the family of all crosscuts of

$$
G_{1}:=\left\{z=\alpha_{j}+r e^{i \theta}: \beta_{j}-\alpha_{j}<r<2\left(\beta_{j}-\alpha_{j}\right), 0<\theta<\pi\right\}
$$


which join $F_{1}:=K \cap\left[\beta_{j}, 2 \beta_{j}-\alpha_{j}\right]$ with $\left[3 \alpha_{j}-2 \beta_{j}, 2 \alpha_{j}-\beta_{j}\right]$. Since $\Gamma_{1}$ is "fewer and longer" than $\Gamma$, the comparison principle yields

$$
m\left(\Gamma_{1}\right) \leq m(\Gamma)
$$

Note that by (1.5),

$$
\operatorname{cap}\left(F_{1}\right) \geq \lambda_{K}\left(\beta_{j}-\alpha_{j}\right)
$$

Consider the conformal mapping of $G_{1}$ onto

$$
G_{2}:=\left\{w=r e^{i \theta}: r_{0}<r<1,0<\theta<\pi\right\}, \quad r_{0}:=\exp \left(-\frac{\pi^{2}}{\log 2}\right)
$$

given by the function

$$
w=f(z):=\exp \left(\frac{i \pi}{\log 2} \log \frac{z-\alpha_{j}}{\beta_{j}-\alpha_{j}}\right)
$$

with the boundary correspondence

$$
\begin{gathered}
f\left(\left[\beta_{j}, 2 \beta_{j}-\alpha_{j}\right]\right)=\left\{w=e^{i \theta}: 0 \leq \theta \leq \pi\right\}, \\
f\left(\left[3 \alpha_{j}-2 \beta_{j}, 2 \alpha_{j}-\beta_{j}\right]\right)=\left\{w=r_{0} e^{i \theta}: 0 \leq \theta \leq \pi\right\} .
\end{gathered}
$$

Since for $\beta_{j} \leq x_{1}<x_{2} \leq 2 \beta_{j}-\alpha_{j}$,

$$
\left|f\left(x_{2}\right)-f\left(x_{1}\right)\right| \geq \frac{x_{2}-x_{1}}{2\left(\beta_{j}-\alpha_{j}\right)},
$$

by the Fekete-Szegö Theorem (see [23, p. 153]) and (4.9) for the set $F_{2}:=f\left(F_{1}\right)$ we have

$$
\operatorname{cap}\left(F_{2}\right) \geq \frac{\operatorname{cap}\left(F_{1}\right)}{2\left(\beta_{j}-\alpha_{j}\right)} \geq \frac{\lambda_{K}}{2}
$$

Furthermore, let $\Gamma_{2}:=f\left(\Gamma_{1}\right)$ and denote by $\Gamma_{3}$ the family of all crosscuts of the annulus $\left\{\tau: r_{0}<|\tau|<1\right\}$ which join $F_{3}:=F_{2} \cup \overline{F_{2}}$, where $\overline{F_{2}}:=\left\{\tau: \bar{\tau} \in F_{2}\right\}$, with the circular boundary component $\left\{\tau:|\tau|=r_{0}\right\}$. By the symmetry principle $m\left(\Gamma_{3}\right)=2 m\left(\Gamma_{2}\right)$. Now we apply Pfluger's theorem (see [22, p. 212]) to obtain

$$
\begin{aligned}
m\left(\Gamma_{3}\right) & \geq \pi\left(\log \frac{1+r_{0}}{\sqrt{r_{0}} \operatorname{cap}\left(F_{3}\right)}\right)^{-1} \\
& \geq \pi\left(\log \frac{1+r_{0}}{\sqrt{r_{0}} \operatorname{cap}\left(F_{2}\right)}\right)^{-1} \geq \pi\left(\log \frac{2\left(1+r_{0}\right)}{\sqrt{r_{0}} \lambda_{K}}\right)^{-1}=: 2 c_{3} .
\end{aligned}
$$

Therefore, the conformal invariance of the module yields

$$
m\left(\Gamma_{1}\right)=m\left(\Gamma_{2}\right)=\frac{1}{2} m\left(\Gamma_{3}\right) \geq c_{3},
$$


which together with (4.8) implies (4.7).

At last, by virtue of the conformal invariance of the module, as well as (4.6) and (4.7), we have (4.5) with $c_{2}:=c_{3}^{-1}$.

Let now $1 \leq N<\infty$. According to [34], for $n \in \mathbf{N}$, either $\Phi_{\Omega}(z)^{n}$ is singlevalued or it is multiple-valued. In the first case, we set $W_{n}(z):=\Phi_{\Omega}(z)^{n}$ and in the second case there exist $q \leq N$ points $x_{1, n}, \ldots, x_{q, n} \in I \backslash K$, such that

$$
W_{n}(z):=\Phi_{\Omega}(z) \prod_{l=1}^{q} \Phi_{\Omega}\left(z, x_{l, n}\right)^{-1}, \quad z \in \Omega,
$$

is single-valued in $\Omega$. According to [34, pp. 159, 211] each complementary interval $\left(\beta_{j}, \alpha_{j+1}\right)$ cannot have more than one point from $\left\{x_{l, n}\right\}$.

Let polynomials $F_{n}(z)=F_{n}(z, K)$ be defined as in Section 2, i.e.,

$$
F_{n}(z):=\frac{1}{2 \pi i} \int_{C_{n}} \frac{W_{n}(\zeta)}{\zeta-z} d \zeta, \quad z \in \mathbf{C},
$$

where $C_{n} \subset \Omega \backslash\{\infty\}$ is a Jordan curve, oriented in the positive direction, containing $K$ and $z$ in its interior.

By the Cauchy formula, for $z \in \Omega \backslash\{\infty\}$ and sufficiently small $t>0$, we have

$$
F_{n}(z)=W_{n}(z)+\frac{1}{2 \pi i} \int_{\tilde{K}_{t}} \frac{W_{n}(\zeta)}{\zeta-z} d \zeta,
$$

where $\tilde{K}_{t}:=\{\zeta \in \Omega: d(\zeta, K)=t\}$ consists of $N+1$ disjoint curves each surrounding exactly one component of $K$.

Passing to the limit, we obtain for $z \in \Omega$ with $|z|<2$,

$$
\begin{aligned}
\left|F_{n}(z)\right| & \leq\left|\Phi_{\Omega}(z)\right|^{n}+\frac{1}{2 \pi} \lim _{t \rightarrow 1^{+}} \int_{\tilde{K}_{t}} \frac{\left|\Phi_{\Omega}(\zeta)\right|^{n}}{|\zeta-z|}|d \zeta| \\
& \leq e^{n g_{\Omega}(z)}+\frac{1}{\pi} \int_{I \backslash D(z, d(z, K)} \frac{|d \zeta|}{|\zeta-z|} \\
& \preceq e^{n g_{\Omega}(z)}+|\log d(z, K)| .
\end{aligned}
$$

Here,

$$
D(z, r):=\{\zeta:|\zeta-z|<r\}, \quad z \in \mathbf{C}, r>0 .
$$

According to (4.2) and (4.10), for $z$ with the property $g_{\Omega}(z)=1 / n$, we have the inequality

$$
\left|F_{n}(z)\right| \leq c_{4} \log (n+1), \quad c_{4}=c_{4}\left(\lambda_{K}\right),
$$


which by the maximum principle for $F_{n}$ in $K_{1 / n}$ is also true for $z \in K$.

Note that $F_{n}(z)=\alpha_{n} z^{n}+\ldots$, where as in $(2.2)$

$$
\begin{aligned}
\left|\alpha_{n}\right| & =\lim _{z \rightarrow \infty}\left|\frac{F_{n}(z)}{z^{n}}\right|=\lim _{z \rightarrow \infty}\left|\frac{W_{n}(z)}{z^{n}}\right| \\
& =\operatorname{cap}(K)^{-n} \exp \left(-\sum_{l=1}^{q} g_{\Omega}\left(x_{l, n}\right)\right) .
\end{aligned}
$$

Therefore, by (4.4),

$$
t_{n}(K) \leq\left\|\frac{F_{n}}{\alpha_{n}}\right\|_{K} \operatorname{cap}(K)^{-n} \leq c_{4} \log (n+1) \exp (V(K)),
$$

where

$$
V(K):=\sum_{j=1}^{N} v_{j}
$$

and $v_{j}=v_{j}(K)$ are defined by (4.3).

Proof of Theorem 3. Applying linear transformation if necessary we always can assume that $K$ satisfies (4.1). By virtue of Theorem 2, the only nontrivial case arises when $K$ consists of infinitely many components. Consider

$$
K_{n}^{*}:=I \cap\left\{z \in \mathbf{C}: g_{\Omega}(z) \leq 1 / n\right\}, \quad n \in \mathbf{N} .
$$

It is worth pointing out that $K_{n}^{*}$ is uniformly perfect with $\lambda\left(K_{n}^{*}\right)=\lambda(K)$. Moreover, by Lemma $3, K_{n}^{*}$ consists of $N+1=N(K, n)+1 \leq c_{5} n$ disjoint closed intervals and

$$
\operatorname{cap}(K) \leq \operatorname{cap}\left(K_{n}^{*}\right) \leq \operatorname{cap}\left(\left\{z \in \mathbf{C}: g_{\Omega}(z) \leq 1 / n\right\}\right)=e^{1 / n} \operatorname{cap}(K)
$$

Let $F_{n}(z)=F_{n}\left(z, K_{n}^{*}\right)$ be the Faber-Widom polynomial as above (constructed for $K_{n}^{*}$ instead of $\left.K\right)$. Denote by $v_{n, j}:=v_{j}\left(K_{n}^{*}\right), j=1, \ldots, N$, the quantities $v_{j}$ defined by (4.3) for $K_{n}^{*}$ instead of $K$. Note that

$$
\max _{1 \leq j \leq N} v_{n, j} \leq \sup _{1 \leq j<\infty} v_{j}(K)=c_{6}
$$

For sufficiently large $n$, consider the sets

$$
\begin{gathered}
\Lambda_{0}:=\left\{j: v_{n, j} \leq \frac{1}{n}\right\} \\
\Lambda_{k}:=\left\{j: \frac{2^{k-1}}{n}<v_{n, j} \leq \frac{2^{k}}{n}\right\}, \quad k=1, \ldots, k_{0}:=\left\lfloor\log _{2}\left(n c_{6}\right)\right\rfloor+1 .
\end{gathered}
$$


Since the number of elements in $\Lambda_{0}$ is at most $c_{5} n$ and by Lemma 3 the number of elements in $\Lambda_{k}$ is at most $c_{7} n 2^{-k}$, we obtain

$$
V\left(K_{n}^{*}\right)=\sum_{k=0}^{k_{0}} \sum_{j \in \Lambda_{k}} v_{n, j} \leq c_{5}+c_{7} k_{0} \leq c_{8} \log n .
$$

Therefore, by (4.11) and (4.12)

$$
t_{n}(K) \leq t_{n}\left(K_{n}^{*}\right) \frac{\operatorname{cap}\left(K_{n}^{*}\right)^{n}}{\operatorname{cap}(K)^{n}} \preceq n^{c 8} \log n,
$$

which implies (1.6).

Acknowledgements. Part of this work was done during the Fall of 2016 semester, while the author visited the Katholische Universität Eichstätt-Ingolstadt and the Julius Maximilian University of Würzburg. The author is also grateful to F. Nazarov for his helpful comments.

\section{References}

[1] L. V. Ahlfors, Lectures on Quasiconformal Mappings, Van Nostrand (Princeton, N. J., 1966).

[2] V. V. Andrievskii, The highest smoothness of the Green function implies the highest density of a set, Ark. Mat., 42 (2004), 217-238.

[3] V. V. Andrievskii, Polynomial approximation of polyharmonic functions on a complement of a John domain, J. Approx. Theory, 190 (2015), 116-132.

[4] V. V. Andrievskii, Chebyshev polynomials on a system of continua, Constr. Approx., 43 (2016), 217-229.

[5] V. V. Andrievskii and H.- P. Blatt, Discrepancy of Signed Measures and Polynomial Approximation, Springer-Verlag (Berlin/New York, 2002).

[6] A. F. Beardon and Ch. Pommerenke, The Poincaré metric of plane domains, J. London Math. Soc. 18 (1978), 475-483.

[7] L. Carleson, On $H^{\infty}$ in multiply connected domains, Conference on harmonic analysis in honor of Antoni Zygmund, Vol. II, ed. Becker, W., et al, Wadsworth, (1983), 349-372. 
[8] J. S. Christiansen, B. Simon, and M. Zinchenko, Asymptotics of Chebyshev polynomials, I. Subsets of $\mathbf{R}$, to appear in Acta Math. Sze.

[9] J. Clunie, On schlicht functions, Ann. of Math., 69 (1959), 511-519.

[10] V. K. Dzjadyk, Introduction to the Theory of Uniform Approximation of Functions by Polynomials, Nauka (Moscow, 1977) (Russian).

[11] D. Gaier, The Faber operator and its boundedness, Journal of Approximation Theory, 101 (1999), 265-277.

[12] J. B. Garnett and D. E. Marshall, Harmonic Measure, Cambridge University Press (New York, 2005).

[13] A. Goncharov and B. Hatinoglu, Widom factors, Potential Anal., 42 (2015), 671-680.

[14] Jahresbericht Deutsch. Math. Verein, (1971) 73:1-5.

[15] D. S. Jerison and C. E. Kenig, Boundary behavior of harmonic functions in non-tangentially accessible domains, Adv. in Math., 46 (1982), 80-147.

[16] O. Lehto and K. I. Virtanen, Quasiconformal Mappings in the Plane, 2nd ed., Springer-Verlag (New York, 1973).

[17] B. Ya. Levin, Majorants in classes of subharmonic functions. I-III, in Teor. Funktsii, Funktsional. Anal. i Prilozhen., 51 (1989), 3-17; 52 (1989), 3-33 (Russian).

[18] F. Peherstorfer and P. Yuditskii, Asymptotic behavior of polynomials orthonormal on a homogeneous set. Journal D'Analyse Math., 89 (2003), 113154 .

[19] Ch. Pommerenke, On the coefficients of close to convex univalent functions, J. London Math. Soc., 41 (1966),161-165.

[20] Ch. Pommerenke, Problems in complex function theory, Bull. London Math. Soc., 4 (1972), 354-366.

[21] Ch. Pommerenke, Uniformly perfect sets and the Poincaré metric, Arch. Math. (Basel) 32 (1979), 192-199.

[22] Ch. Pommerenke, Boundary Behaviour of Conformal Maps, Springer-Verlag (Berlin/New York, 1992).

[23] T. Ransford, Potential Theory in the Complex Plane, Cambridge University Press (Cambridge, 1995). 
[24] E. B. Saff and V. Totik, Logarithmic Potentials with External Fields, Springer-Verlag (Springer-Verlag, 1997).

[25] V. I. Smirnov and N. A. Lebedev, Functions of a Complex Variable. Constructive Theory, Mass. Institute of Technology (Cambridge, 1968).

[26] M. L. Sodin and P. M. Yuditskii, Functions least deviating from zero on closed subsets of the real line, St. Petersburg Math. J., 4 (1993), 201-249.

[27] P. K. Suetin, Series of Faber Polynomials, Gordon and Breach Science Publishers (Amsterdam, 1998).

[28] P. M. Tamrazov, Smoothnesses and Polynomial Approximations, Naukova Dumka (Kiev, 1975) (Russian).

[29] V. Totik, Chebyshev polynomials on a system of curves. Journal D'Analyse Mathématique, 118 (2012), 317-338.

[30] V. Totik, Chebyshev polynomials on compact sets. Potential Anal., 40 (2014), 511-524.

[31] V. Totik, Asymptotics of Christoffel functions on arcs and curves, Advances in Mathematics, 252 (2014), 114-149.

[32] V. Totik and T. Varga, Chebyshev and fast decreasing polynomials, Proc. London Math. Soc., published online March 25, 2015.

[33] J. L. Walsh, Interpolation and Approximation by Rational Functions in the Complex Plane, fith ed., American Mathematical Society (Providence, 1969).

[34] H. Widom, Extremal polynomials assosiated with a system of curves in the complex plane, Adv. Math., 3 (1969), 127-232. 